EPJ Web of Conferences 19, 06003 (2012)

DOI: $10.1051 /$ epjconf/20121906003

(C) Owned by the authors, published by EDP Sciences, 2012

\title{
The formation of the Galactic bulge of the Milky Way
}

\author{
M. Ness ${ }^{\mathrm{a}}$ and K. Freeman
}

Research School of Astronomy \& Astrophysics, The Australian National University

\begin{abstract}
We aim to determine if the bulge formed via mergers as predicted by Cold Dark Matter (CDM) theory, or from disk instabilities, as suggested by its boxy shape, or both processes. We are observing about 28,000 bulge stars in fields that span longitudes of -31 to $+26^{\circ}$ and latitudes of $-5^{\circ}$ to $-10^{\circ}$, targeting mostly red clump giants and we are measuring stellar velocities and chemical abundances. We have almost concluded our observations and have analysed data of 23,000 stars. We find a cylindrical rotation profile for the bulge which blends smoothly out into the disk and from the $[\mathrm{Fe} / \mathrm{H}]$ results we find the bulge to be comprised of separate components, with an underlying slowly rotating metal poor subsample which we believe to be the inner halo stars and metal weak thick disk. We find only a small $[\mathrm{Fe} / \mathrm{H}]$ gradient with latitude in the bulge, of $-0.07 \mathrm{dex} / \mathrm{kpc}$. This weak gradient does not necessarily support a merger origin for our bulge and the composite nature of the bulge is consistent with formation out of the thin disk as per instability formation models.
\end{abstract}

\section{INTRODUCTION}

Galactic bulges are relevant to galaxy formation as they are signatures of formation and we see two primary classes of bulges in our universe, large classical bulges and smaller boxy bulges. The aims of our study are to determine if the Milky Way bulge was formed via mergers (classical bulges), or disk instability (boxy bulges) and to achieve our aims we are undertaking a spectroscopic survey at medium resolution in order to characterise the metallicity and kinematics of the bulge. We are examining the relationship between kinematics and metallicity and comparing our data to models, to look for signatures of formation.

\section{SIGNATURES OF FORMATION}

The signatures of formation between classical merger generated bulges and disk instability boxy bulges are quite distinct. For the kinematics, boxy bulges show a cylindrical rotation profile, with the bulge rotating as rapidly at high latitudes as it does in the plane and this has been seen both observationally [1] and in simulations [2]. Classical bulges however, show a spheroidal rotation profile, with slower rotation at high latitudes. In the chemistry, boxy bulges should show common properties between bulge stars and thin and thick disk stars, as theory proposes that boxy bulges are actually bars seen edge on, and formed out of the disk. Classical bulges should have a larger metallicity gradient, due to the rapid star formation and also CDM predicts that the first stars will be found in the central regions of the galaxy, ie. the bulge [3].

a e-mail: mkness@mso.anu.edu.au

This is an Open Access article distributed under the terms of the Creative Commons Attribution-Noncommercial License 3.0, which permits unrestricted use, distribution, and reproduction in any noncommercial medium, provided the original work is properly cited. 


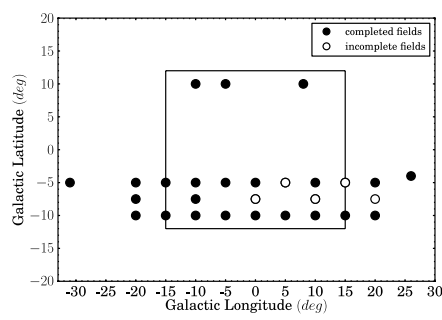

(a) $R_{\text {gal }}<3.5 \mathrm{kpc}$

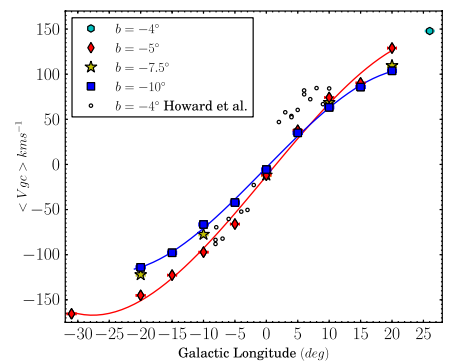

(b) $R_{\text {gal }}<3.5 \mathrm{kpc}$

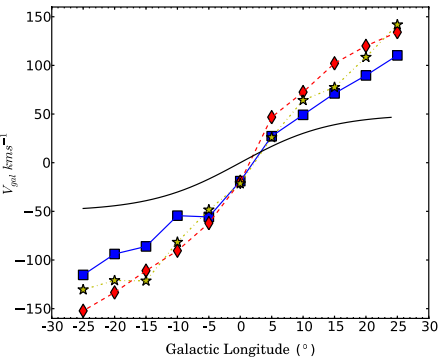

(c) $R_{\text {gal }}<3.5 \mathrm{kpc}$

Figure 1. In (a) our fields surveyed; In (b) our rotation curve for bulge stars at $R_{\text {gal }} \leq 3.5 \mathrm{kpc}$ at $b=-5^{\circ},-10^{\circ}$; In (c) the rotation curve of the N-body model at $b=-5^{\circ}, b=-10^{\circ}$, where we also show in the solid black line a spheroidally rotating classical bulge at $b=-10^{\circ}$.

\section{THE MILKY WAY BULGE}

The Milky Way bulge is relatively small, with a boxy/peanut bar/bulge morphology, about $8 \mathrm{kpc}$ away and inclined by $25^{\circ}$ with respect to the line of sight [4]. Our survey of the bulge is of about 28,000 stars and our fields are shown in Figure 1(a) where the bulge is represented by an inner rectangle. Our stars are selected from the infra red survey catalogue 2MASS and we are selecting primarily for red clump giants, as these have a well defined absolute magnitude, enabling distance determinations; $M_{K}=-1.65$, $\left.\sigma=0.2,(J-K)_{0}=0.65\right)[5]$.

\section{OBSERVATIONS AND ANALYSIS}

We have observed our stars on the $3.9 \mathrm{~m}$ Australian Anglo Telescope using the AAOmega $2 \mathrm{dF}$ spectrograph. Our primary region for analysis covers from 8400 - 8850 Angstroms at a resolution of $\mathrm{R} \sim 11,000$. Radial velocities, to $\leq 1.2 \mathrm{~km} \mathrm{~s}^{-1}$, have been determined using the dominant Ca II triplet lines in the spectra. The $[\mathrm{Fe} / \mathrm{H}]$ abundance and $[\alpha / F e]$ has been determined via a $\chi^{2}$ program we have written for this dataset, based on the method of Kirby et al. 2008 [6], where Fe lines have been used for the $[\mathrm{Fe} / \mathrm{H}]$ abundance and a combination of $17 \mathrm{Ti}, \mathrm{Si}$ and $\mathrm{Mg}$ lines have been used for determining $[\alpha / \mathrm{Fe}]$. The synthetic spectral library for this been generated from the Castelli Kurucz model atmospheres [7] using the Stellar line sythesis program MOOG [8].

\section{RESULTS}

The stellar parameters have been determined for all 23,000 stars observed to date to an accuracy of $T_{\text {eff }} \pm 75 \mathrm{~K}, \log \mathrm{g} \pm 0.35,[\mathrm{Fe} / \mathrm{H}] \pm 0.13,[\alpha / \mathrm{Fe}] \pm 0.1$. We have determined distances to each star in our survey using Basti isochrone (10 Gyrs) fits and red clump magnitudes and find $60 \%$ of the stellar sample remains in $R_{g a l} \leq 3.5 \mathrm{kpc}$, the cylindrical galactocentric radius. These stars span a metalicity of -2.6 to 0.6 with almost $50 \%$ of the stars in the $[\mathrm{Fe} / \mathrm{H}]$ range -0.5 to 0 .

The rotation curves of the bulge are shown in Figure 1(b). These curves are consistent with a cylindrical rotation profile for the bulge and compare favourably with the N-body model of cylindrical rotation provided by our collaborator Lia Athanassoula (private communication), see Figure 1(c). Each marker on our rotation curve corresponds to the mean velocity of about 850 stars and the rotation curves blend smoothly out into the disk as predicted by the model.

We confirm a metallicity gradient along the minor axis. Figure 2(a) shows our histograms for our three minor axis fields; about 1500 stars in total. The mean $[\mathrm{Fe} / \mathrm{H}]$ goes from $\sim-0.18$ dex at our lowest latitude field to $\sim-0.5 \mathrm{dex}$ at our highest latitude field which corresponds to a gradient in $[\mathrm{Fe} / \mathrm{H}]$ of 


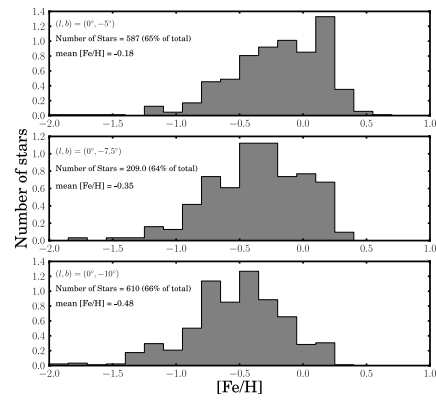

(a) $R_{\text {gal }}<3.5 \mathrm{kpc}$

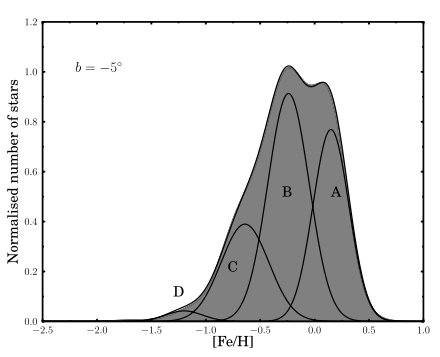

(b) $R_{\text {gal }}<3.5 \mathrm{kpc}$

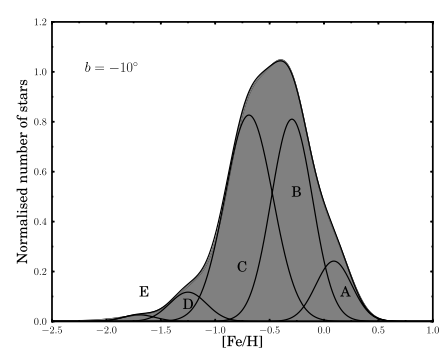

(c) $R_{\text {gal }}<3.5 \mathrm{kpc}$

Figure 2. For $R_{\text {gal }}<-3.5 \mathrm{kpc}$ : In (a) our histograms of our 1500 stars along the minor axis; In (b) and (c), the generalised histograms and components fit for $l= \pm 15^{\circ}$, at $b=-5^{\circ}, b=-10^{\circ}$.

$-0.4 \mathrm{dex} / \mathrm{kpc}$. Our mean $[\mathrm{Fe} / \mathrm{H}]$ is $\sim 0.15$ dex lower than that found by Zoccali et al. (2008) from their high resolution survey $(\mathrm{R} \sim 45,000$ ) of about $850 \mathrm{~K}$ giants and the gradient is also lower by 0.2 dex than their $0.6 \mathrm{dex} / \mathrm{kpc}$ across a latitude of $-4^{\circ}$ to $-12^{\circ}$ degrees.

For our fields across the entire bulge, and within $R_{\text {gal }} \leq 3.5 \mathrm{kpc}$, from a longitude of $-15^{\circ}$ to $+15^{\circ}$, we generate generalised histograms for our latitudes of $-5^{\circ}$ and $-10^{\circ}$, where each data point in $[\mathrm{Fe} / \mathrm{H}]$ is represented by a gaussian with the standard deviation of $\pm 0.13 \mathrm{dex}$, our error in $[\mathrm{Fe} / \mathrm{H}]$. These are shown in Figure 2: (b) and (c). We find that we can fit the same four gaussian components, to both latitudes of observational data and these we call A, B, C, D. There is an additional metal poor component E seen only at the higher latitude. At the lower latitudes, components A and B are dominant and at the higher latitudes, components $\mathrm{B}$ and $\mathrm{C}$ are dominant in the sample. Each component is shifted down in its mean by 0.05 dex at the higher latitude. Considering the bulge to be comprised of these components rather than represented by a single distribution, the metallicity gradient, as measured per component, is $-0.07 \mathrm{dex} / \mathrm{kpc}$.

Looking along radial distance, we measure a gradient in component A only, of approximately $-0.025 \mathrm{dex} / \mathrm{kpc} \pm 0.01$. We believe that component $\mathrm{B}$, which we see in fairly consistent contribution fractions inside the bulge region, at $R_{\text {gal }}<3.5 \mathrm{kpc}$ is the true bar/bulge component, more metal poor than the inner thin disk which may have continued to undergo chemical evolution and corresponds to component $\mathrm{A}$. Component $\mathrm{A}$ decreases with latitude and $\mathrm{C}$ increases and we believe these are the thin and thick disk, respectively, given their relative scale heights of about $300 \mathrm{pc}$ and $700 \mathrm{pc}$ [10]. Components D and E are consistent with the metallicity of the metal weak thick disk and inner halo stars observed in our sample. Figures 3(a) and (b) show the alpha enhancement across the bulge, $R_{\text {gal }} \leq 3.5 \mathrm{kpc}$, longitudes $\pm 15^{\circ}$ and latitudes $-5^{\circ}$ and $-10^{\circ}$. The corresponding gaussian components A,B,C,D are included. All components except for component A are alpha enhanced. Due to the lower contribution fraction of the metal rich stars at higher latitudes, more of the stars at higher latitudes are alpha enhanced.

Finally we examine the rotation as a function of the kinematics. To isolate the metal poor component of the sample, we made a cut in $[\mathrm{Fe} / \mathrm{H}]$ of $\leq-1.0$ and $>-1.0$. The rotation curve of stars $[\mathrm{Fe} / \mathrm{H}] \leq$ -1.0 is shown in Figure 3(c). The lower metallicity stars represent only $5 \%$ of the data and these stars still demonstrate a net rotation that is consistent, at the higher latitude, with a spheroidal rotation profile. At the higher latitude, the velocity remains flat out into the disk where as at the lower latitude the velocity increases to the same level as for the stars in the metal rich cut, at a longitude of $-20^{\circ}$ and $+15^{\circ}$. This is likely due to the sampling of a higher fraction of metal weak thick disk, compared to dispersion supported halo stars at the lower latitudes, as seen in the generalised histograms of the components. 


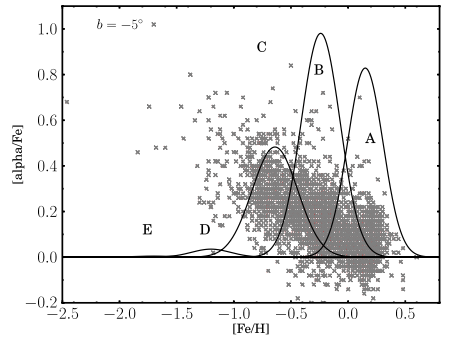

(a) $R_{g a l}<3.5 \mathrm{kpc}$

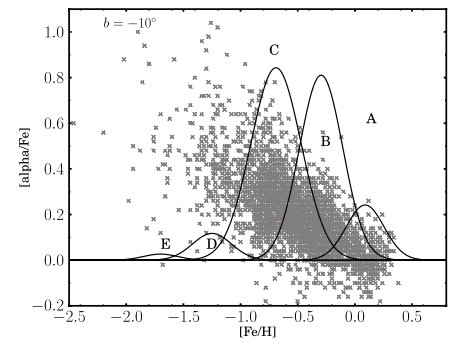

(b) $R_{g a l}<3.5 k p c$

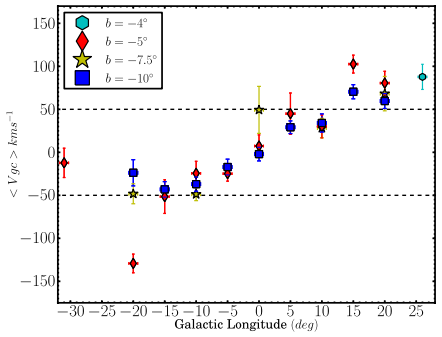

(c) $R_{g a l}<3.5 \mathrm{kpc}$

Figure 3. $[\alpha / \mathrm{Fe}]$ at $b=-5^{\circ}$ in (a) and at $b=-10^{\circ}$ in (b) shown with components; (c) shows the metal poor rotation, for stars $[\mathrm{Fe} / \mathrm{H}] \leq-1.0$.

\section{DISCUSSION}

We find that the bulge is a composite system and this interpretation has important implications for the signatures of formation. The large metallicity gradient measured across latitude, of $-0.6 \mathrm{dex} / \mathrm{kpc}$ has been argued as evidence for a merger generated origin of the bulge (Zoccali et al. 2008). However, the smaller gradient that we find, per component of $-0.07 \mathrm{dex} / \mathrm{kpc}$, does not necessarily support this scenario. We ascribe our sample to components A-E; as thin disk, bar/bulge/thick disk/metal weak thick disk and inner halo.

The bulge rotation curve is cylindrical and blends smoothly out into the disk, as predicted by the $\mathrm{N}$-body instability models for bulge formation out of the thin disk. Following on from this scenario, the bulge should be more metal poor than the inner thin disk today, which has continued to undergo chemical evolution. Effectively, the metallicity of the bulge is a marker of the dynamical timescale and age at which the buckling process took place in the galaxy. That the bulge and thick disk are alpha enhanced, although at a lower $[\mathrm{Fe} / \mathrm{H}]$ range for the bulge, may be evidence for a common origin and a marker of the different times and timescales of spatial redistribution for these components. It is likely that most of the stars in this $[\mathrm{Fe} / \mathrm{H}]<-1.0$ cut are metal weak thick disk and inner halo stars on highly eccentric orbits passing through the bulge region but we do not exclude an underlying merger generated component at this time.

\section{CONCLUSIONS}

We have determined all stellar parameters of $T_{e f f},[\mathrm{Fe} / \mathrm{H}],[\alpha / \mathrm{Fe}]$ and $\log \mathrm{g}$ for each star and the bar/bulge membership from this. We find the bulge to be cylindrically rotating out into the disk and find the bulge to be a composite system. These populations consist of, in order of decreasing metallicity, the thin disk, the true bulge/bar, the thick disk and metal weak thick disk and halo. We confirm a metallicity gradient with latitude but attribute this as being due to the different contribution fractions of the different components. We see a weak gradient with radial distance in component $\mathrm{A}$, the thin disk, only. We find rotation velocity decreases with $[\mathrm{Fe} / \mathrm{H}]$ (and dispersion increases) and we see a slowly rotating metal poor component at $[\mathrm{Fe} / \mathrm{H}] \leq-1.0$ which we believe is the halo and metal weak thick disk stars in our sample. We will search for more clues of formation from individual abundances (Ti,Si,Mg,Al,O,Ca) and $\mathrm{N}$-body models. All data to date are consistent with the instability scenario for bulge/bar formation with the bulge/bar being component $\mathrm{B}$ with a mean $[\mathrm{Fe} / \mathrm{H}]$ of -0.24 and formed out of the early thin disk.

\section{References}

[1] Kormendy, J. and Kennicutt, Jr., A\& A, 42, 2004, 603-683.

[2] Athanassoula, E., MNRAS, 2005, 358, 1477-1488 
[3] Diemand, J. and Madau, P. and Moore, B., MNRAS, 2005, 364, 367-383

[4] Gerhard, O., ASPC, 273, 2002, 73.

[5] Pietrzyński, G. and Gieren, W. and Udalski, A. AJ, 2004,125, 2494-2501.

[6] Kirby, E. ApJ, 2008, 682, 1217-1233

[7] Castelli, F. and Kurucz, R. L.astro-ph, 2004, 5087C

[8] Sneden, C. and Lambert, D. L. MNRAS, 1975, 170, 533-540.

[9] Zoccali, M., Hill, V., Lecureur, A., Barbuy, B., Renzini, A, Minniti, D., Gómez, A., Ortolani, S., A\&A, 2008, 79, 177-189

[10] Van der Kruit, P. C. and Freeman, K.C., arXiv, 2001, 1101.1771 